\title{
Relación del nivel de conocimiento y práctica del Modelo SOAPIE en la Calidad de las anotaciones de los profesionales de Enfermería del Hospital II EsSalud, Vitarte
}

\author{
Karen Liliana CASTILLO ASENCIOS*
}

EAP de Enfermería, Facultad de Ciencias de la Salud, Universidad Peruana Unión, Alt. Km 19; Carretera Central, Lurigancho, Lima

\begin{abstract}
RESUMEN
El objetivo del estudio fue determinar la relación del nivel de conocimiento y práctica sobre el modelo SOAPIE en la calidad de las anotaciones de los profesionales de enfermería del Hospital II EsSalud de Vitarte. La investigación fue de tipo cuantitativo de diseño no experimental de corte transversal y de alcance descriptivo, correlacional, teniendo como muestra 30 enfermeras y 90 anotaciones de enfermería. Los instrumentos aplicados fueron un cuestionario sobre el modelo SOAPIE y una lista de chequeo que evalúa la práctica del modelo y la calidad de las anotaciones en base al Proceso Atención Enfermería. Los resultados fueron que el conocimiento y práctica en el modelo SOAPIE se relaciona en mínimo porcentaje con la estructura de las anotaciones de enfermería, asimismo el nivel de conocimiento se relaciona débilmente con el contenido de estas a diferencia de la práctica donde la relación es muy fuerte en el contenido de las anotaciones.
\end{abstract}

Palabras clave: Anotaciones de enfermería; proceso atención de enfermería (PAE); calidad; modelo SOAPIE.

\begin{abstract}
The aim of this study was to determine the relationship of the level of knowledge and practice model SOAPIE on the quality of the annotations of the nursing staff of Hospital II EsSalud Vitarte. The quantitative research was non-experimental design and scope transversal descriptive, correlational. The sample included 30 nurses and 90 nursing notes. The instruments used were a questionnaire on the SOAPIE model and a checklist that evaluates SOAPIE practice model and the quality of the entries based on SAP. We found that knowledge and practice in the SOAPIE model relates to minimum percentage in the structure of the nursing notes, also the knowledge level is related weakly to the content of those opposed to the practice where the relationship is very strong in the content of the notes.
\end{abstract}

Keywords: Nursing notes, nursing care process (NCP), quality, model SOAPIE

*correspondencia: peardusty.upeu.edu.pe, 51-1-976918363 


\section{INTRODUCCIÓN}

En la actualidad existe diversidad de registros que son utilizados por el personal de enfermería, los cuales tienen normas que guían su elaboración; sin embargo, a pesar de ser un documento legal y de servir como documentos de auditoría, existen deficiencias en cuanto a su elaboración en su contenido. Las Anotaciones de Enfermería son registros en el cual se plasman los acontecimientos más importantes de lo sucedido y realizado al paciente que permite la evolución del cuidado, valorar las acciones que realiza la enfermera y si éstas están de acuerdo a las necesidades del paciente tomándolo como un ser individual con necesidades enmarcadas en diversos áreas como lo físico, psicológico, social y espiritual, asimismo constituye un documento legal, ya que respalda las acciones de la enfermera(o) frente a la atención del paciente en la institución laboral (Fretel, 2004).

La documentación existente sobre los registros de enfermería a nivel internacional resalta la necesidad de contar con ellos como parte fundamental del crecimiento profesional. Alconedo, Fernández, Pérez, y Sola (2000), realizaron un estudio donde se valoró los registros de enfermería de 40 pacientes portadores de marcapaso temporal, al analizar los resultados llegaron a la conclusión que existía un porcentaje elevado de registro deficientes en datos importantes. El registro de enfermería es parte de la actividad diaria de la enfermera (o) y el reflejo del trabajo principal de nuestra profesión, el cuidado del paciente (Díaz, García, Lobo, \& Ruiz, 2001).

Los registros clínicos de enfermería forman parte de los expedientes de cualquier institución hospitalaria, de esta manera se registran las intervenciones que desarrolla enfermería en su labor asistencial, de acuerdo a las necesidades que presenta cada paciente (Rivas, Martínez, Ibáñez, Aguilera, \& Sánchez, 2009). Los hospitales tienen la autoridad de definir sus propios registros de enfermería, siempre y cuando estos registros cumplan las normas legales y profesionales autorizadas (Ibarra \& García, 2007). La Joint Comission on Acreditation of Healthcare Organizations (JCAHO) en su manual de acreditación para hospitales que establece niveles de cumplimiento especificos que cada institución debe crear sus propios niveles de cumplimiento de acuerdo a su filosofía, cultura de calidad y organización. En este sentido, propone estándares internacionales cuyo propósito es adaptar su modelo de acreditación a otros países. Dichos estándares se elaboraron con la intensión de que tengan un grado óptimo y que a la vez sean alcanzables, pues solo así pueden propiciar un compromiso tangible en torno a la calidad de atención. (Ortega, Suárez, Jiménez, Anorve, Cruz M., \& Cruz G., 2009).
Ibarra y García (2007), mencionan que la administración del cuidado de enfermería y los registros clínicos de enfermería deben cumplir las normas de la profesión y apoyarse para su desempeño en la American Nurses Asociation (ANA) y en la JCAHO, organizaciones que poseen normas para el registro de enfermería, a nivel mundial, las cuales establecen las responsabilidades del personal de enfermería, por las cuales se puede juzgar la calidad de la práctica del servicio. Una de las normas que establece la JCHAJO para la documentación de enfermería es que los registros de enfermería se deban basar en normas establecidas en el SOAPIE, ya que en este modelo, los cuidados de enfermería se basan en los diagnósticos de enfermería identificados por la NANDA. (“Asociación Nacional para la Clasificación de los Diagnósticos de la Enfermería Americana”).

El panorama a nivel nacional con respecto al ámbito internacional no difiere mucho, la mayoría de los documentos referentes a este tema, destacan los aspectos que deben contener los registros y la importancia de los mismos, hoy en día constituye un desafío contar con un registro de enfermería eficaz que evidencie la continuidad del cuidado y promover una garantía de calidad en la atención del usuario.

El Código de Ética y Deontología del Colegio de Enfermeras (os) del Perú en el Capítulo III, Artículo $28^{\circ}$ indica que los registros de Enfermería deben ser claros, precisos, objetivos sin enmendaduras y realizados por la Enfermera (o) que brinda el cuidado debiendo registrar su identidad. (Código de Ética y Deontología del Colegio de Enfermeras).

Lo expuesto permitió identificar a la investigadora el deficiente conocimiento y práctica del modelo SOAPIE en la calidad de las anotaciones de las enfermeras del Hospital II de Vitarte, originado muchas veces por la deficiente actualización de las enfermeras en anotaciones de enfermería en el modelo SOAPIE. Asimismo, Anglade (2006), menciona que la capacitación continua se debe considerarse como función importante de la institución empleadora de trabajadores de salud, ya que ésta constituye un factor importante que condiciona la calidad y el desempeño laboral, además ayuda a mantener la satisfacción en el empleo. Si hablamos que la capacitación es importante en todos los niveles, esto cobra mayor importancia puesto que el personal profesional debidamente capacitado se constituye en un elemento de cambio; cuya participación en la atención que proporciona a los pacientes está acompañada de un sustento teórico que respalda su quehacer diario, aunque estudios realizados mostraron que, a pesar de que las enfermeras conozcan y relaten respecto a la importancia de sus anotaciones para la continuidad del cuidado, de que éstas 
se constituyan en un documento legal, de que subsidien el trabajo de otros profesionales, ellas continúan produciendo registros incompletos y carentes de significado (Oliveira \& Cadette, 2009).

También la elaboración de las anotaciones de enfermería se encuentran asociados a los factores personales e institucionales, evidenciando que en la población éstos desfavorecen (70\%) su elaboración destacando los indicadores interés personal y sobrecarga de trabajo respectivamente. Trayendo como consecuencia un registro de enfermería donde existe limitación de información sobre la condición del paciente o acciones a cumplir en el turno laboral subsiguiente a entregas de guardias con informaciones rutinarias resumidas, ambiguas, carente de información a los progresos alcanzados por el paciente, a interpretaciones erróneas debido a su poca profundidad y extensión para posibilitar la continuidad del plan de atención o cumplimiento de acciones delegadas e independientes por el incumplimiento de registro preciso. (Gutiérrez \& Cuerva, 2006).

Por todo lo expuesto anteriormente, se hace necesario investigar esta área tan indispensable, no solo para el profesional de enfermería y su gestión, sino como evidencia de la calidad del cuidado que se brinda al usuario, con el propósito de incentivar el perfeccionamiento de las habilidades cognoscitivas de las enfermeras y la mejora de la práctica de las anotaciones de enfermería. La investigación se trazó como objetivo determinar la relación del nivel de conocimiento y práctica sobre el modelo SOAPIE en la calidad de las anotaciones del personal de enfermería del Hospital II EsSalud de Vitarte.

\section{MATERIAL Y MÉTODOS}

La investigación fue de tipo cuantitativo de diseño no experimental de corte transversal y de alcance descriptivo El muestreo fue no probabilístico a criterio del investigador; la muestra según criterios de inclusión fue de 30 enfermeras de los servicios de Medicina, Cirugía, UVI, Pediatría y Emergencia. Los instrumentos utilizados fueron: Cuestionario para evaluar conocimientos sobre los registros de enfermería en especial sobre el modelo SOAPIE. Y una lista de chequeo dividido en dos dimensiones estructura y contenido de las anotaciones de enfermería según el modelo SOAPIE.

\section{RESULTADOS}

\section{Tabla 1}

Conocimientos sobre el modelo de SOAPIE de los profesionales de Enfermería del Hospital II de Vitarte.

\begin{tabular}{ccc}
\hline Nivel de conocimientos & $\mathrm{N}$ & $\%$ \\
\hline Muy malo & 2 & 6,7 \\
Malo & 10 & 33,3 \\
Regular & 9 & 30,0 \\
Bueno & 7 & 23,3 \\
Muy bueno & 2 & 6,7 \\
Total & 30 & 100,0 \\
\hline
\end{tabular}

En la tabla 1, se observa que el $33.3 \%$ de los profesionales de enfermería presenta nivel malo de conocimientos sobre el modelo de SOAPIE, 30\% nivel regular y solo el $6.7 \%$ muy bueno.

Tabla 2

Tipos de registro de anotaciones de las Enfermeras del Hospital II de Vitarte según el modelo elegido

\begin{tabular}{ccc}
\hline Tipos de registro & $\mathrm{N}$ & $\%$ \\
\hline Narrativo & 20 & 66,7 \\
SOAPIE & 10 & 33,3 \\
Total & 30 & 100,0 \\
\hline
\end{tabular}

En la tabla 2, se observa que el 66.7\% utiliza el modelo narrativo y solo el 33.3\% utiliza el modelo SOAPIE. Cabe recalcar que la respuesta a esta pregunta fue corroborada al momento de evaluar las anotaciones de enfermería. 
Tabla 3

Nivel de práctica del modelo SOAPIE en las anotaciones de Enfermería del Hospital II de Vitarte

\begin{tabular}{ccc}
\hline Nivel de práctica del modelo SOAPIE & N & $\%$ \\
\hline Muy malo & 4 & $10 \%$ \\
Malo & 4 & $10 \%$ \\
Regular & 10 & $40 \%$ \\
Bueno & 8 & $30 \%$ \\
Muy bueno & 4 & $10 \%$ \\
Total & 30 & $100 \%$ \\
\hline
\end{tabular}

Con respecto a la práctica del modelo SOAPIE en las anotaciones de enfermería, solo se evaluó las anotaciones realizadas con este modelo, donde el $40 \%$ presenta nivel de práctica regular y el 30\% nivel bueno, asimismo el 10\% presenta nivel de práctica muy malo, malo y muy bueno respectivamente.

Tabla 4

Nivel de estructura de las anotaciones de Enfermería del Hospital II de Vitarte

\begin{tabular}{ccc}
\hline Nivel de estructura & $\mathrm{N}$ & $\%$ \\
\hline Muy malo & - & - \\
Malo & - & - \\
Regular & - & $100 \%$ \\
Bueno & 30 & - \\
Muy bueno & - & $100 \%$ \\
Total & 30 & \\
\hline
\end{tabular}

En la tabla 4, se aprecia la primera dimensión evaluada, la estructura de las anotaciones y tuvo como resultado que el $100 \%$ presento nivel bueno.

Tabla 5

Nivel de contenido de las anotaciones de Enfermería del Hospital II de Vitarte

\begin{tabular}{ccc}
\hline Nivel & $\mathrm{N}$ & $\%$ \\
\hline Muy malo & 22 & $24,4 \%$ \\
Malo & 40 & $44,4 \%$ \\
Regular & 13 & $14,4 \%$ \\
Bueno & 14 & $15,6 \%$ \\
Muy bueno & 1 & $1,1 \%$ \\
Total & 90 & $100 \%$ \\
\hline
\end{tabular}

La tabla 5 muestra la segunda dimensión que es el contenido de las anotaciones, donde el 44.4\% presentó un nivel malo y solo el 1.1\% presentó un nivel muy bueno; asimismo entre el 14\% y 15\% presenta nivel regular y bueno respectivamente. 
Tabla 6

Nivel de calidad de las anotaciones de Enfermería del Hospital II de Vitarte

\begin{tabular}{ccc}
\hline Nivel de calidad & $\mathrm{N}$ & $\%$ \\
\hline Malo & 25 & $27,8 \%$ \\
Regular & 39 & $43,3 \%$ \\
Bueno & 22 & $24,4 \%$ \\
Muy bueno & 4 & $4,4 \%$ \\
Total & 90 & $100 \%$ \\
\hline
\end{tabular}

En total del nivel de calidad obtenido promedio es regular con el $43.3 \%$ y solo el $4.4 \%$ obtuvo un nivel de calidad muy bueno; asimismo el $27.8 \%$ y el $24.4 \%$ presentan nivel de calidad malo y bueno respectivamente.

Tabla 7:

Consolidación de los datos correlaciónales

\begin{tabular}{ccccc}
\hline \multicolumn{1}{c}{ Correlación entre } & $\mathrm{R}$ & Sig. & $\mathrm{R} 2$ & Decisión \\
\hline $\begin{array}{c}\text { Nivel de conocimiento y práctica en } \\
\text { el modelo SOAPIE sobre el nivel } \\
\text { de estructura de las anotaciones de } \\
\quad \text { enfermería }\end{array}$ & 0,426 & 0,49 & 0,18 & $\begin{array}{c}\text { Existe correlación } \\
\text { positiva débil }\end{array}$ \\
$\begin{array}{c}\text { Nivel de conocimiento y práctica en el } \\
\text { modelo SOAPIE y nivel de contenido } \\
\text { de las anotaciones de enfermería }\end{array}$ & 0,997 & $0,000 * *$ & $\begin{array}{c}0,993 \\
\text { Relación=99,3\% }\end{array}$ & $\begin{array}{c}\text { Existe correlación } \\
\text { positiva muy fuerte }\end{array}$ \\
$\begin{array}{l}\text { Nivel de conocimiento y práctica en el } \\
\text { modelo SOAPIE y nivel de calidad de } \\
\text { las anotaciones de enfermería }\end{array}$ & 0,995 & $0,000^{* *}$ & $\begin{array}{c}0,990 \\
\text { Relación=99\% }\end{array}$ & $\begin{array}{c}\text { Existe correlación } \\
\text { positiva muy fuerte }\end{array}$ \\
\hline ** La correlación es significativa al nivel 0,01 (bilateral). & & &
\end{tabular}

\section{DISCUSIÓN}

Condezo (2008), refiere que los cambios cognitivos puede darse en el comportamiento, como en el aumento de desempeño de algún trabajo, lo que difiere de los resultados donde se hallaron los niveles de conocimiento de las enfermeras es de malo a regular con el $33.3 \%$ y $30 \%$ respectivamente; también menciona que la retroalimentación es la esencia del proceso llamado refuerzo, en el caso de los profesionales las actualizaciones constantes o las asistencias a seminarios incrementa o mejora lo que se conoce con nuevas innovaciones en la materia de especialización optimizando el trabajo que se realiza; mientras que los resultados obtenidos demuestran que el 26.6\% de las enfermeras no asistió a ningún seminario de actualización y que el 50\% solo asistió a un seminario durante todo el año 2010.

Por otro lado, entre el nivel de práctica del modelo SOAPIE y la calidad de la estructura existe relación débil, aunque se halló relación altamente significativa en la calidad del contenido de las anotaciones de enfermería de lo cual se infiere que la aplicación del modelo SOAPIE influye en la calidad de las anotaciones de enfermería, demostrado por los resultados obtenido en el nivel de calidad de aquellas enfermeras que realizaron anotaciones con el modelo SOAPIE en concordancia con los resultados encontrados por Pecho (2003), quien realizó un programa donde se introduce el modelo SOAPIE en la realización de las notas de enfermería, halló que el 5\% presentó nivel excelente en el pre test mientras que en el post test el $25 \%$ presentaba nivel de calidad excelente, demostrando que existe relación significativa entre la aplicación del modelo SOAPIE y la calidad de las anotaciones de enfermería.

Al respecto Iyer \& Tapich (1997), señala que las siglas SOAPIE son variaciones comunes del formato SOAP original y siguen en forma más completa la aplicación del proceso de atención de enfermería, proporcionando un registro fijo en la evolución del paciente de los 
métodos empleados por la enfermera, para llegar a un diagnóstico de cuidados y decidir los procedimientos para proporcionar los cuidados y evaluar la eficacia de la terapia asistencial. Según Cortez y Castillo (2009), SOAPIE es un método sistemático para el registro de calidad por la demanda de pacientes, la rutina o por la falta de una capacitación permanente.

En la investigación también se halló que en la calidad de las anotaciones de enfermería el 33.3\% presentó nivel malo y el 30\% nivel regular, estos resultados son similares a Anglade (2006), quien observó que un porcentaje representativo de las Anotaciones de Enfermería revisadas se catalogan como no significativas, concluyendo que la atención brindada en estas anotaciones no fue integral, evidenciado por la escasa cantidad de registros. Fretel (2004), también realizó una investigación evaluando las anotaciones de enfermería durante los años 2000-2002 del HNGAI, concluyendo que el $50 \%$ son medianamente significativas, siendo solo el $25 \%$ significativas, lo cual evidencia que el cuidado brindado reflejado no ha sido integral ya que el contenido de las anotaciones de enfermería revisadas solo se basan en términos generales sobre el estado del paciente; en cuanto a los criterios tomados en la evaluación de las anotaciones encontró que solo el 21\% es calificada como adecuada, $56.6 \%$ regular y el $21.8 \%$ no adecuada, lo cual indica que en más de la mitad de la población en estudio no considera entre sus criterios de elaboración datos importantes en base al PAE.

Pozo, Guerra, \& Álvarez (2005), refieren que a pesar de los esfuerzos realizados para la incorporación del Proceso de Atención de Enfermería, como método de trabajo, se ha observado que presenta dificultades al llevarlo a la práctica tanto por los estudiantes como los profesionales en muchos otros países donde se enseña y exige, por considerarlo un método conveniente y vá- lido pero complejo en su ejecución. También refieren que actualmente su aplicación está limitada principalmente, porque el personal de enfermería enmarca su trabajo en dar cumplimiento a las indicaciones médicas con pobre actuación independiente, y no ocupa en el equipo de salud el lugar que le corresponde.

Donabedian (1993), refiere que la búsqueda de la calidad constituye la dimensión ética de loa vida profesional, en efecto los propios profesionales deben ser los primeros interesados en mantener la calidad personal y colectivamente en sus actuaciones, en el campo de su competencia. Así, el Consejo Internacional de Enfermeras (CIE) incluye en su Código Ético la obligación del establecimiento y garantía de altos estándares en la práctica y los conocimientos de las enfermeras de todo el mundo.

\section{CONCLUSIONES}

- En relación con los objetivos e hipótesis planteados se establece lo siguiente:

- Respecto a la relación que tiene el Nivel de conocimiento y práctica en el modelo SOAPIE sobre el Nivel de estructura de las anotaciones de enfermería la correlación es positiva débil, por lo que se aprueba la hipótesis nula y se rechaza la alterna.

- Con respecto al Nivel de conocimiento y práctica en el modelo SOAPIE sobre el Nivel de contenido de las anotaciones de enfermería la correlación es positiva muy fuerte; por lo tanto, se aprueba la hipótesis alternativa y rechazando la nula.

- De acuerdo a los resultados anteriores, podemos decir que la relación entre el Nivel de conocimiento y práctica en el modelo SOAPIE sobre el Nivel de calidad de las anotaciones de enfermería la correlación es positiva muy fuerte; por lo tanto, se aprueba la hipótesis alterna y se rechaza la nula. 


\section{REFERENCIAS BIBLIOGRÁFICAS}

1. Alconedo, A., Fernández, R., Pérez, S., \& Sola, J. (2000). Registro de enfermería y marcapasos temporal transvenoso. Recuperado de http://dialnet. unirioja.es/servlet/busquedadoc?db=1\&t=Registr os + de + enfermer $\% \mathrm{C} 3 \% \mathrm{ADa}+\mathrm{y}+$ marcapasos + tem poral+transvenoso\&td=todo.

2. Anglade, C. (2006). Características de las anotaciones de enfermería y factores personales e institucionales asociados a su elaboración en el Hospital Nacional “Dos de Mayo”. Recuperado de http://www.cybertesis.edu.pe/sisbib/2006/anglade_vc/pdf/anglade_vc.pdf.

3. Código de Ética y Deontología (2008). Estatutos y reglamento ley de trabajo de la enfermera. Recuperado de http://www.cepjunin.com/Codigo_etica.pdf.

4. Condezo, M. H. (2008). La enseñanza aprendizaje y la anotaciones de enfermería para una función profesional eficiente. Recuperado de http://www. cybertesis.edu.pe/sisbib/2008/condezo_mm/pdf/ condezo_mm-TH.3.pdf.

5. Consejo Nacional del Colegio de Enfermeros del Perú (2008). Normas de gestión de la calidad del cuidado enfermero.

6. Cortez, G., \& Castillo, F. (2009). Guía para elaborar proceso, registros, protocolo y cuidado de enfermería. Ed. Rodhas.

7. Consejo Internacional de Enfermeras. (2006). Recuperado de http://www.icn.ch/images/stories/documents/about/icncodespanish.pdf

8. Díaz, P., García, J., Lobo, A., \& Ruiz, M. (2001). Control y registro, por enfermería de la tensión arterial en el episodio anginoso. Recuperado de Instituto de cardiología de Madrid: http://dialnet. unirioja.es/servlet/articulo? codigo $=2330990$.

9. Fretel, V. R. (2004). Evaluación de las anotaciones de enfermería durante los años 2000-2002 en el servicio de neurología del Hospital Nacional Gui- llermo Almenara Irigoyen. Recuperado de http:// www.cybertesis.edu.pe/sisbib/2004/fretel_pv/pdf/ fretel_pv.pdf.

10. Gutiérrez, M., \& Cuerva, A. (2003). Evolución en los registros de los cuidados de enfermería en los niños intervenidos de hipospadias. Recuperado el 15 de diciembre de 2010, de http://www.semes. org/revista/vol18_4/7.pdf

11. Ibarra, A. J., \& García, M. d. (2007). Registros de enfermería. Recuperado de http://www.eccpn.aibarra.org/temario/seccion1/capitulo21/capitulo21. htm.

12. Iyer, P., \& Tapich, B. (1997). Proceso y diagnóstico de enfermería (7ma ed.). México: Gra-Hill. Interamericana.

13. Oliveira, V., \& Cadette, M. (2009). Anotaciones del enfermero en el acompañamiento del crecimiento y desarrollo infantil. Recuperado el 16 de diciembre de 2010, de http://www.scielo.br/scielo.php?pid=S0103$21002009000300010 \& \mathrm{script}=\mathrm{sci}$ abstract\&tlng=es

14. Pecho, M. (2003). Efectividad del programa educativo en el mejoramiento del conocimiento y calidad de las anotaciones de enfermería en el Hospital Regional de Ica. Recuperado de http://www. cybertesis.edu.pe/sisbib/2003/pecho_tm/pdf/pecho_tm.pdf.

15. Pozo, E., Guerra, E., \& Álvarez, L. (2005). Recuperado de http://bvs.sld.cu/revistas/ems/vol15_1_01/ ems04101.htm

16. Rivas, J., Martínez, F., Ibáñez, D., Aguilera, M., \& Sánchez, L. (2009). Propuesta de registros clínicos de enfermería con vinculación taxonómica para úlceras, traqueostomía y estomas. Recuperado de http://www.imbiomed.com. $\mathrm{mx} / 1 / 1 /$ articulos.php? method=showDetail\&id articulo $=59694 \&$ id_seccion $=702 \&$ id ejemplar $=6037 \&$ id_revista $=41$ 